\title{
ON COLLIMATION OF THE OUTFLOWS IN FORCE-FREE
}

\section{MAGNETOSPHERES}

\author{
I. OKAMOTO \\ National Astronomical Observatory \\ Mitaka, Tokyo, 181 Japan \\ e-mail: okamoto@nao.ac.jp
}

The "force-free" (FF) approximation has often been evoked as a convenient means of treating electromagnetic processes of extracting rotational energy from pulsars and black holes through their magnetospheres, to energize a variety of activities in the environments. In order to produce relativistic jets with cylindrical topology like those associated with AGNs, some people further use this approximation for collimating relativistic outflows in the asymptotic domains from central sources. It is argued here that one can not collimate outflows by the FF magnetic field only, that is, the flow-field structure must asymptotically be conical far out from the light cylinder.

The magnetosphere under concern is described by a so-called pulsar equation for the flux function $P$,

$$
\nabla \cdot\left[\frac{1}{\varpi^{2}}\left(1-\frac{\alpha^{2} \varpi^{2}}{c^{2}}\right) \nabla P\right]+\frac{1}{2 \varpi^{2}} \frac{d \beta^{2}}{d P}+\frac{\alpha^{2}}{c^{2}} \frac{d \ln \alpha}{d P}|\nabla P|^{2}=0
$$

and the poloidal and toroidal components of the electromagnetic field are expressed as

$$
\boldsymbol{B}_{\mathrm{p}}=-\frac{\boldsymbol{t} \times \boldsymbol{\nabla} P}{\varpi}, \quad \boldsymbol{B}_{\mathrm{t}}=\frac{\beta(P)}{\varpi} \boldsymbol{t}, \quad \boldsymbol{E}_{\mathrm{p}}=-\frac{\alpha(P)}{c} \nabla P, \quad \boldsymbol{E}_{\mathrm{t}}=0
$$

(Michel 1973a,b; Scharlemann \& Wagoner 1973; Julian 1973; Okamoto 1974). These expressions contain the two functions of $P$, i.e. $\alpha(P)$ for the angular velocity of field lines and $\beta(P)$ for the angular momentum flux per unit flux tube. Usually $\alpha$ is given at the feet of field lines, while $\beta$ is often regarded as a free function of $P$. We show that since the toroidal component of the field is a swept-back component of the poloidal field by plasma particles, $\beta$ is not freely specifiable even in the $\mathrm{FF}$ approximation, 
but should be determined by loading particle inertia on field lines at the 'sphere at infinity', where plasma inertia is thrown away;

$$
\beta(P)=-\frac{\alpha(P)}{c}\left(B_{P} \varpi^{2}\right)_{\infty} .
$$

We refer to this condition as the plasma condition on the $\mathrm{FF}$ magnetosphere. This condition is also equivalent to the condition given by taking the FF limit of the criticality condition at the magnetosonic point in the relativistic centrifugal winds.

The asymptotic behavior of the pulsar equation for $\varpi \gg \varpi_{\mathrm{L}} \equiv c / \alpha$ is described from Eq. (1) by

$$
\frac{1}{r^{2}} \frac{\partial}{\partial r}\left(r^{2} \frac{\partial P}{\partial r}\right)-\frac{1}{2 \varpi^{2}} \frac{\partial}{\partial P}\left(\varpi \frac{\partial P}{\partial r}\right)^{2}=\frac{c^{2}}{2 \alpha^{2} \varpi^{2}} \frac{\partial}{\partial P}\left[\beta^{2}-\left(\frac{\alpha}{c} B_{\mathrm{P}} \varpi^{2}\right)^{2}\right]
$$

where $\partial / \partial P \equiv\left(1 / B_{\mathrm{P}}^{2} \varpi^{2}\right)(\nabla P \cdot \nabla)$.

If $\partial P / \partial r=0$, i.e. $P=P(\theta)$ in the asymptotic domain, one has $B_{P} \varpi^{2}=$ $\sin \theta(d P / d \theta)$ and then the right hand side of Eq. (4) reduces to condition (3). Conversely condition (3) requires the field structure to be of $P=P(\theta)$ in the asymptotic domain. That is, the field tends to be radial, i.e. conical toward infinity, filling all the space there. Thus as far as we retain the FF approximation, the FF field must become radial asymptotically and cannot by itself collimate toward the axis.

It can be shown that condition (3) indicates Ohm's law for the surface return current $\mathcal{I}_{\infty}$ on the 'sphere at infinity' with surface resistivity $R_{\mathrm{L}}=$ $4 \pi / c=377 \mathrm{Ohm}$. Actually from Eq. (3) one has $\mathcal{I}_{\infty}=(c \beta / 4 \pi \varpi)_{\infty} \boldsymbol{e}_{\theta}=$ $\left(1 / R_{\mathrm{L}}\right) \boldsymbol{E}_{\theta}$, where $\boldsymbol{E}_{\theta}=(\beta / \varpi)_{\infty} \boldsymbol{e}_{\theta}$. This ensures the global current closure.

In derivation of $\beta(P)$ in Eq. (3), we had to impose the condition of $v \rightarrow c$, to make the force-free approximation break down at $\varpi \gg \varpi_{\mathrm{L}}$, and to restore the inertia to massless charged particles. This leads automatically to breakdown of the frozen-in condition, and finite electric conductivity enables charged particles to cross the radial field lines on the sphere at infinity, following Ohm's law. The resulting Joule dissipation of the surface electric current equals the total Poynting flux flowing into the sphere at infinity. One can interpret this situation as conversion of the electromagnetic energy extracted from the central object to the kinetic energy of accelerated nonmassless particles beyond the force-free region.

Thus the FF field cannot self-collimate toward the rotation axis, unless there is any external confining mechanism (see Okamoto, I., Astronomy and Astrophysics, 326, issue 3 (October III, 1997), pp. 1277-1284 for details). 